\title{
XIX.
}

Aus der k. k. chirurgischen Universitätsklinik des Herrn Hofrathes Professor Dr. C. Nicoladoni in Graz.

\section{Zur Verwendung der Quetschmethoden in der Darmchirurgie.}

Von

Dr. Erwin Payr,

Privatdocent für Chirargie und Assistent obiger Klinik.

(Mit 2 Abbildungen.)

Die Quetschmethoden haben sich in der Darmchirurgie der Jetztzeit einen hervorragenden Platz gesichert. Das von Souligoux ${ }^{1)}$ zuerst verwendete, dann von Doyen ${ }^{2}$ verallgemeinerte und techniseh zu hoher Vollkommenheit gebrachte Verfahren hat schnell Anerkennung gefunden (Lanz $\left.{ }^{3}\right)$. Die deutschen Chirurgen haben sich erst in allerneuester Zeit allgemeiner dem Verfahren zugewendet und ist dies besonders der Empfehlung Mikulicz'4) und seiner Schüler zuzuschreiben.

Das Enterotrib v. Mikuliez', das in so überaus bequemer Weise "gestattet, beliebige Partieen des Magen-Darmkanals durchzuquetschen und durch den Schnürverschluss bleibend zu vereinigen, ist heutzutage zweifellos ein viel gebrauchtes und sehr zweckmässiges Instrument.

Besonders dort, wo man eine seitliche Einpflanzung eines Darmstückes vornimmt, ermöglicht es in schnellster und bequemster Weise den blinden Verschluss des axialen freien Endes.

1) Souligoux, Gastro-enterostomie par un nouveau procédé. France mêd. 1896. Nr. 29.

2) Doyen, Eine neue Methode derPylorus- u. Darmresection. Verhandlungen der deutschen Gesellschaft f. Chirurgie. 1898.

3) Lanz, Die Quetschmethoden im Dienste der Magenchirurgie; Beiträge zur klin. Chirurgie. XXX.Bd. 3. Heft.

4) v.Mik uli cz, Handbuch der prakt. Chirurgie (mit K a u s c h) III. Bd. I. Hälfte. 
Das Instrument ist mehrfach zu diesem Zwecke von uns mit völlig befriedigendem Erfolge verwendet worden. - Besonders häufig wurde es aber bei der Abtragung des Wurmfortsatzes benützt; der nach Ligatur der sehr verdünnten Quetschfurche zurückbleibende kleine Stumpf lässt sich ja sehr leicht und schnell versenken und übernähen.

Bei Gelegenheit der Entfernung mehrerer acut oder subacut entzündeter Wurmfortsätze machte ich die Beobachtung, dass das Quetschinstrument in Folge der entzündlich veränderten Beschaffenheit der Appendixwand diese völlig durchtrennt und nach Abnahme des Instrumentes nur einige Gewebsfasern statt der erwarteten Serosamanchette übrig geblieben sind. In solchen Fällen kann man denken, dass durch die schwere Erkrankung am Wurmfortsatze die Gewebe so brü chig sind, dass bei starker Quetschung ein völliges Auseinanderweichen aller Gewebschichten zu Stande kommt und auch die Serosa nicht mehr die Fähigkeit des Widerstandes hat.

Bekanntlich sollen ja alle Schichten des Darmes, besonders die Mucosa, aber auch die Muskelschichten völlig durchgequetscht werden, während die Serosa und eventuell die Submucosa als die widerstandsfähigsten erhalten bleiben.

Als Hauptvortheil neben der Reinlichkeit des Verfahrens ist wohl auch die sehr bequeme Art der Blutstillung angesehen worden und soll ja der Akt der Durchquetschung jede isolirte Gefässunterbindung überflüssig machen.

Es giebt aber doch Fälle, in denen die Anwendung der Qu etsehmethoden (Enteprotrib) nicht angezeigt ist und zu unangenehmen Vorkommnissen führen kann; ich glaube durch die Mittheilung einiger darauf Bezug habender Erfahrungen zeigen zu können, dass es selbst für diese so einfache und schön erdachte Technik - wenn auch in sehr engen Grenzen - Contrain dicationen giebt, die selbstverständlich den Werth der Methode nicht schmälern sollen, noch können.

Ich glaube, es ist für eine Methode im Gegentheil besser, wenn sie in einem Falle, in dem sienicht ganz sichere Resultate geben kann, nicht zur Anwendung kommt.

Nachfolgende kurze Krankengeschichte möge das, was ich beabsichtige, erläutern und zugleich einen kleinen Beitrag zn dem stets wieder Ueberraschungen bietenden Capitel der H e rni e n l e h re bringen.

19 jährige Frau wird am 26. October 1901 mit seit 6 Tagen eingeklemmter, faustgrosser, rechtsseitiger Cruralhernie aufgenommen. 
Schlechtes Allgemeinbefinden; trockene Zunge, frequenter kleiner Puls; ca. $125-130$.

Verfallenes Aussehen, fortwährend Erbrechen grülicher Massen und Singultus.

Temperatur 39,9. Abdomen stark aufgetrieben, in beiden Flanken etwas freie Fltussigkeit. Druckempfindlichkeit des Bauches nicht besonders gross. Die Haut uber dem Bruche zeigt leichte Entzündungserscheinungen.

Bei der $11 / 2$ Stunden nach der Aufnahme der Patientin vorgenommeneu Operation in Aethernarkose wird als Bruchinhalt eine ca. $15 \mathrm{~cm}$ lange Dünndarmschlinge und ein Netzssttlck vorgefunden, Nach Erweiterung der Bruchpforte lässt sich die zufturende Darmschlinge weit hervorziehen, während die abfuhrende vollkommen fixirt er-

Anlöthung des untersten Ileums an's Cöcum.

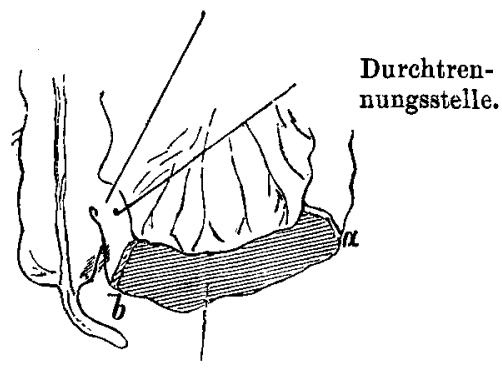

Gangränöse Dünndarmschlinge, a, b, Schnürfurchen

Fig. 1. scheint. Die Schnurfurchen sind gangränös, der eingeklemmte Darm ist an einer Stelle seiner Convexität perforirt. Um die Ursache der Fixation des abfuhrenden Darmstuckes kennen zu lernen, wird das Poupart'sche Band in der Richtung nach oben weit eingeschnitten und ergiebt sich nun, dass die eingeklemmte Darmschlinge vom letzten Theil des Ileum gebildet ist; bei kräftigem Anziehen am abführenden Darm. stücke erscheint das Cöcum mit dem Processus vermiformis in der Bruchpforte. Die Gangrän erfordert die Resection der Darmschlinge, welche aber an Ort und Stelle nicht ausführ-

bar ist, da die Schnturfurche am abfuhrenden Schenkel der Schlinge kaum zwei Querfinger von der Einmundung des Ileums in das wenig beweg. liche Cöcum entfernt ist.

Deshalb wird der Hautschnitt nach aufwärts und gegen die Spina anterior superior verlängert und die Bauchwand oberhalb des Poupartschen Bandes in einer Ausdehnung von ca. $8 \mathrm{~cm}$ durchtrennt. Der undurchsehnittene Theil des Poupart'schen Bandes und der Musculatur bleiben als Brücke zwischen dem Laparotomieschnitt und dem Bruchgebiete stehen. Die Dunndarmschlinge wird sorgfältig in Jodoformgaze eingehullt, durch den Bruchring zurickgeschoben und durch die neu angelegte Bauchdeckenwunde heranggezogen.

Resection eines ca. $16 \mathrm{~cm}$ langen Darmstückes knapp an der Einmüdungsstelle in das Cöcum. Bei dieser Gelegenheit zeigt es sich nun, dass das unterste Ileumstuck nicht unter einem rechten Winkel, sondern unter einem spitzen in den Dickdarm einmündet und mit seiner dem Mesenterialansatze gegenuber liegenden Seite mit der medialen Wand des cöcalen Blindsackes anf ein Stück weit innig verlöthet war; es bestand an dieser Stelle eine relative Stenose des untersten Ileums und wäre eine circuläre Naht unter Berücksichtigung der angedenteten Verhältnisse nicht 
nur schwierig, sondern auch unzweckmässig gewesen (Fig. 1.) Es war beabsichtigt, das kleine, nach der Resection noch am Cöcum thrig bleibende Ileumstück mit der Quetschmethode und Schnürmethode blind zu verschliessen und das centrale Ileumstück an einer Stelle der Vorderwand des Cöcums einzupflanzen (End-zu-Seit-Anastomose). Während dieses Operationsactes waren folgende Beobachtungen zu machen:

Das abzuquetschende Darmstiick ist trotz einer Entfernung von $\mathbf{c a} .3 \mathrm{~cm}$ von der distalen Schniurfurche etwas succulent und sein Peritoneum leicht

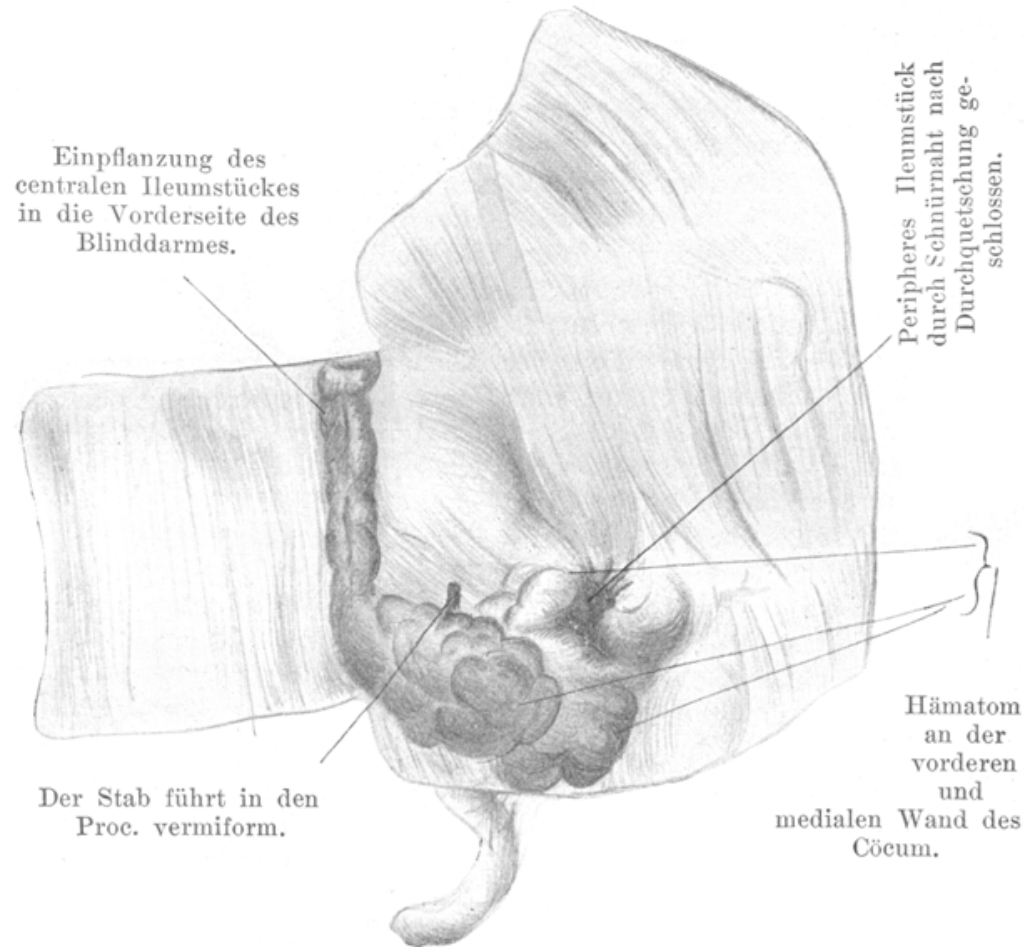

Fig. 2.

injicirt. Als das lege artis angelegte Enterotrib nach genitgend langer Dauer der Quetschung abgenommen wurde, trat an mehreren Stellen der Schnürfurche hellgelber, ganz dünnflüssiger, übelriechender Darminhalt an die Oberfläche, und man sah deutlich, dass an einzelnen Stellen auch die Serosablätter durchgequetscht waren.

Ausserdem bildet sich nahe an der Schnärfurche ein $\mathrm{Hämatom}$ aus, das in kürzester Zeit die Wand des am Cöcum hängenden Darmstückchens und den Blinddarm stark auf blähte. Ich bemerke ansdrücklich, dass bei der Resection der Dünndarmschlinge die Mesenterialgefässe in Portionen sorgfältig bis an die Durchtrennungsstelle nahe am Blinddarm abgebunden waren und es sich nur um die Stillung der Blutung aus dem 
Schnittrande des Darmes handelte, die eben nach dem Doyen'schen Verfahren durch den Zangendruck zugleich mit dem Darmverschluss geschehen soll.

Mittelst einer Ligatur wurde der Darm knapp am Rande einer neu angelegten Schnittfurche abgebunden und der Stumpf nach der tiblichen Behandlung durch eine fortlaufende Lembertnaht versenkt (s. Fig. 2).

Nunmehr wurde nach dem Nahtvorgange Kocher's ${ }^{1}$ ) das centrale Darmstück in die Vorderwand des Cöcums eingepflanzt. Nach Eröffnung des Blinddarmes sah ich eine mächtige, wurstartige Blutgeschwulst unter der Schleimbat des Cöcums sich von der verschlossenen Stelle des Darmes gegen die Vorderseite zu ausbreiten. Das Hämatom war mindestens $1 \mathrm{~cm}$ dick und gut daumenbreit und reichte dis zur medialen Anastomosennaht.

Trotz dieses ziemlich mächtigen Hämatoms wurde rasch die laterale Nahtreihe beendet - die neugebildete Communication war sehr weit - und wurde an den Verschluss der Bauchdeckenwunde gegangen. Ueber die Resectionsstelle und ther die vordere und mediale Wand des Cöcums wird ein kleiner Mikulicz schleier ausgebreitet, im Uebrigen die Laparotomiewunde durch Etagennaht geschlossen.

Das durch die Perforation der Darmschlinge schwer veränderte Bruchbett wird excidirt, der Bruchsack durch Tabakbentelnaht geschlossen, das Poupart'sche Band durch Seidenknopfnähte wieder vereinigt, das Bruchbett mit Gummirohr und Jodoformgazestreifen drainirt. - Im Uebrigen die Haut darüber ebenfalls geschlossen. - Verband.

Am 26. October nach Verabreichung eines Sennaeinlaufes reichliche Stuhlentleerung, kein Erbrechen, Zunge trocken, Puls 120.

27. October. Verbandwechsel. Reichliche Durchfeuchtung. Patientin hat eine gelbliche Gesichtsfarbe. Respiration sehr beschleunigt. Abdomen ganz leicht aufgetrieben, sehr druckempfindlich.

Puls klein, sehr frequent, ab und zu unregelmässig. Sensorium zeitweise getrubt.

28. October. Puls 150-170. Deutliche Zeichen einer septischen Allgemeininfection ohne peritoneale Reizerscheinungen. $800 \mathrm{ccm}$ Kochsalzlösung subcutan. Strophantus, Sauerstoffeinathmung, Campherinjection.

Am 29. Oetober Mittags Exitus letalis.

Aus dem Sectionsbefunde seien nur kurz folgende Bemerkungen hervorgehoben :

Am Dnterlappen der rechten Lunge lobulärpneumonische Herde, in beiden Lungen acutes Oedem. Trübungen des Endocards, beginnende arteriosklerotische Veränderungen an den grossen Gefässen. Nur in der rechten Fossa iliaca findet man an einem Couvolut unterer Ileumsschlingen leichte Verklebungen, stärkere Gefässinjection, jedoch nirgends fibrinöse Beläge. Wenig freie Flüssigkeit. Das Ileum findet sich in das untere Colon ascendens und Cöcum eingenäht; die circuläre Darmnaht vollständig geschlosseu und rein. Das unterste Stück des Ileums, welches nach der Resection ubrig geblieben war, ist am Cöcum zu einem Blindsacke geschlossen. Auch diese Naht zeigt vollkommen reinen Verschluss.

1) Kocher, Chirurgische Operationslehre. 3. Auflage. 
Entsprechend diesem Blindđarmsacke beginnt ein submucöses Hämatom, das sich bis zur circulären Darmnaht erstreckt und ein wurstförmiges Gebilde darstellt.1) Das Peritoneum des Ileums ist nur in der Gegend der erwähnten Darmnähte etwas geröthet, zeigt jedoch keine fibrinösen Beläge. Das übrige Peritoneum der Bauchhöhle ist zart und blass. Subacuter Milztumor. Parenchymatöse Degeneration der Organe.

Obductionsdiagnose:

Pneumonia lobularis lobi inferioris dextri; Oedema acutum lobi superioris dextri. - Sepsis.

Der tödtliche Ausgang des Falles war natürlich nicht unerwartet. Es bestand in der Umgebung der eingeklemmten Darmschlinge zweifellos eine, wenn auch circumscripte Peritonitis. Wir fassten dieselbe als eine Peritonitis chemica im Sinne von Tavel ) und $\operatorname{Lanz}^{3}$ ) auf. Ausser einer stellenweise sehr intensiven Injection und Röthung des Peritoneums und einem intraperitonealen Flüssigkeitsergusse war nur ab und zu Fibrin in Form von feinen Membranen auf den Darmschlingen zu sehen. Viel schwerer als diese Toxinperitonitis war die zur Zeit der Operation sicher schon bestehende Toxinämie; die Erscheinungen, unter denen die Patientin zu Grunde ging, waren ja die einer allgemeinen Sepsis.

Am Localbefunde verdienen drei Umstände besonderes Interesse:

1. Die Einklemmung eines relativ so starkfixirten Darmtheiles, wie es in diesem Falle das unterste Ileum war. - Wir zweifeln nieht, dass die betreffende Ileumschlinge schon in früheren Zeiten den typisch wiederkehrenden Inhalt dieses Bruches gebildet hat. Wie wären sonst die spitzwinklige Einmündung in das Cöcum und die durchaus nicht frischen Verklebungen der einander zu sehenden Darmwände zu erklären?

Dabei ist zu bemerken, dass das Cöcum nicht, wie es sonst so häufig bei Hernien der Fall ist, ein längeres Mesocöcum hatte, sondern straff an der hinteren Bauchwand fixirt war; dies der Grund, warum sich der abführende Schenkel der incarcerirten Schlinge nicht entwickeln liess.

2. Die Anwendung des Mikulicz'schen Enterotribs brachte in diesem Falle eine Unannehmlichkeit, indem das Instrument die Darmwand ganz durchquetschte und Darminhalt aus

1) Herr cand. med. Eppinger hatte die Freundlichkeit, das Präparat zu zeichnen, wofür ich ihm bestens danke.

2) Tavel n. Lanz, Ueber die Aetiologie der Peritonitis; Mittbeilungen an Klinikeu u.medicinischen Instituten der Schweiz. 1893. 1. Reihe, 1. Heft.

3) s. Tavel. 
518 XIX. PAYR, Zur Verwendung der Quetschmethoden in der Darmchirurgie.

der Quetschfurche austrat. - Der Zustand dieses Darmstïckes lässt sich nicht annähernd mit dem eines acut entzündeten Wurmfortsatzes vergleichen. - Und trotzdem leistete die Serosa schon bei einem so leichten Grade der Entzündung und Hyperämie nicht mehr den erwarteten Widerstand. ${ }^{1)}$ Im Zusammenhalt mit unserem bei in verschiedenem Grade entzündeten Wurmforts ätzen gemachten Beobachtungen möchten wir der Ansicht sein, dass bei Staung und Entzündungszuständen am Darme die Anwendung des Mikulicz'schen Enterotribs Einschränkungen zu erleiden hat.

3. Die Blutung, die sogleich nach der Durchquetschung der Darmvand eintrat, war ebenfalls ein unvorhergesehenes und unangenehmes Ereigniss. Auch sie legt den Gedanken nahe, dass in ödematösen und entzündeten Geweben der Vorgang der Blutstillung kein so exacter ist, wie dies sonst bei Durchquetschung ron Magen- und Darmtheilen der Fall ist. - Grössere submucöse Hämatome bedingen bekanntlich Ernährungsstörungen der Schleimhaut und sind jedenfalls nicht als vollkommen gleichgültig zu betrachten; (Garrè $\left.{ }^{2}\right)$, Ritter ${ }^{3}$ ), Vanni $\left.{ }^{4}\right)$ u. A.).

Es erscheint uns daher rathsam, bei Hyperämie und Entzündungszuständen am Magen-Darmkanal die Quetschwirkung einer genauen Kritik zu unterwerfen, da unter Umständen ein völliges Durchquetschen aller Darmwandschichten und unvollkommener Verschluss der Blutgefässe zu beobachten sind.

1) Das Enterotrib, das hier verwendet wurde, ist ganz gleichmässig gearbeitet und kann man dies sehr leicht durch Erzeugen einer Quetschfurche auf einem Blatt dicken weissen Papieres, das man dann bei durchfallendem Lichte betrachtet, feststellen.

2) Garrè, Ueber eine eigenartige Form narbiger Darmstenose nach Brucheinklemmung. Beiträge zur klin. Chir. Bd. IX. S. 185.

3) Ritter, Ueber den Einfluss von Traumen auf die Entstehung des: Magengeschwüres. Zeitschr. f. klin. Medicin. Bd. XII. S. 592.

4) Vanni, Ref. Virchow-Hirsch's Jahresbericht 1889. II. Bd. S. 281. 